\title{
NON-ISOMORPHIC 2-PERFECT 6-CYCLE SYSTEMS OF ORDER 13
}

\author{
RebecCA A.H. Gower
}

\begin{abstract}
Running a computer search for new, cyclic, 2-perfect 6-cycle systems of order 13 and constructing the quasigroups which arise from such systems enabled the author to establish that there are at most two such non-isomorphic systems. Then by using two-variable laws of the quasigroups it is shown that there are exactly two non-isomorphic 2-perfect 6-cycle systems of order 13 which are cyclic.
\end{abstract}

It is known that necessary and sufficient conditions for the existence of a 2-perfect 6-cycle system of order $n$ are that $n \equiv 1$ or $9 \bmod 12$ and $n>9$ (Lindner, Phelps and Rodger [2]). Hence the smallest possible order of such a system is 13 . The existence of a 2-perfect 6-cycle system of order 13 is shown by example in [2]. The example is cyclic.

It is obvious that for large $n$ the construction of a 2-perfect 6-cycle system of order $n$ is not unique up to isomorphism but for small $n$ such a result is not obvious. The most likely case for which uniqueness up to isomorphism might occur seemed to be 13 as it is the smallest possible order for such a system. However there is not a unique 2-perfect 6-cycle system of order 13; in fact the main result of this paper is:

THEOREM. There are precisely two cyclic non-isomorphic 2-perfect 6-cycle systems of order 13.

Arriving at this result involved two main steps; in each I used computer programs I have written to speed the work. The first of these steps was an exhaustive search (performed by the computer) for cyclic 2-perfect 6-cycle systems on 13 points. The second step was to compare the new systems produced in the first step with the system in [2] and with each other, so it could be determined which systems were isomorphic to one another. This comparison involved the use of two-variable quasigroup laws.

Received 21 November 1990.

This work was done while I was employed as a research assistant on a project funded by an ARC grant. I wish to thank Dr Sheila Oates-Williams for her advice and help with this paper and also Martin Sharry for supplying the computer program which I used to check whether or not the laws hold in the quasigroups.

Copyright Clearance Centre, Inc. Serial-fee code: 0004-9729/91 \$A2.00+0.00. 


\section{DEFINITIONS AND PRELIMINARIES.}

DEFINITION: A 6-cycle system of order $\boldsymbol{n}$ is a decomposition of the complete graph, $K_{n}$, into a collection of edge disjoint 6-cycles.

The 6-cycle containing edges $v_{1} v_{2}, v_{2} v_{3}, \ldots, v_{5} v_{6}, v_{6} v_{1}$ is denoted by $\left(v_{1}, v_{2}, v_{3}, v_{4}, v_{5}, v_{6}\right)$.

DEFINITION: A 6-cycle system is said to be 2-perfect if the set of 3-cycles formed by taking edges between vertices which are distance 2 apart in the 6-cycles is also an edge disjoint decomposition of $K_{n}$.

So if $\left(v_{1}, v_{2}, v_{3}, v_{4}, v_{5}, v_{6}\right)$ is a 6-cycle of the system then $\left(v_{1}, v_{3}, v_{5}\right)$ and $\left(v_{2}, v_{4}, v_{6}\right)$ are in the set of 3-cycles formed by taking edges between elements distance 2 apart in the 6-cycles. If this distance 2 graph is indeed an edge disjoint decomposition of $K_{n}$ then it is a Steiner triple system of order $n$ (Lindner [3]).

From a 2-perfect 6-cycle system one can derive a quasigroup in the following manner (see [3]). Define $\circ$ on the vertex set $V$ of $K_{n}$ by:

(i) $(\forall v \in V)(v \circ v=v)$;

(ii) $(\forall u, v \in V, u \neq v)(v \circ u=z$ and $u \circ v=w)$ if and only if $(u, v, w, s, t, z)$ is a 6-cycle of the system for some $s$ and $t$.

Such a quasigroup satisfies the two-variable laws,

(i) $(\forall x \in V)(x \circ x=x)$

(ii) $(\forall x, y \in V)((x \circ y) \circ y=x)$;

(iii) $(\forall x, y \in V)((x \circ y) \circ(y \circ[x \circ y])=x \circ(y \circ x))$.

In the example given in [2] of a 2-perfect 6-cycle system of order $13, K_{13}$ has vertex set $\mathbb{Z}_{13}$ and the collection of 6 -cycles is defined as follows: $C_{13}=\{(0,5,2,8,7,9)+i \mid$ $0 \leqslant i \leqslant 12\}$ where $\left(v_{1}, v_{2}, v_{3}, v_{4}, v_{5}, v_{6}\right)+i=\left(v_{1}+i, v_{2}+i, \ldots, v_{6}+i\right)$ with each component reduced modulo 13 .

\section{THE EXHAUSTIVE SEARCH FOR ORder 13, CYCLIC, 2-PERFECT 6-CYCle SYSTEMS}

Using $\mathbb{Z}_{13}$ as a vertex set for $K_{13}$, adjacent vertices of a 6 -cycle can be considered to have a difference of \pm 1 or \pm 2 or $\ldots$ or \pm 6 between them by letting $d_{i}=v_{i+1(\bmod 6)}-$ $v_{i}, 1 \leqslant i \leqslant 6$. If a system is generated cyclically from one 6 -cycle as in the example above, each of the integers 1 to 6 must occur as the absolute value of a difference between adjacent vertices in order to have each edge of $K_{n}$ appear in a 6-cycle.

For example, in the system above, the differences are $+5,-3,+6,-1,+2,+4$.

In order to get a 6 -cycle it is necessary that the sum of the differences is congruent to 0 mod 13. The first part of the search for other order 13 , cyclic, 6 -cycle systems was to find all possible combinations of signs to couple with the integers 1 to 6 such that 
the resultant sum was congruent to $0 \bmod 13$. That is, couple the sign $s_{i}, s_{i} \in\{+,-\}$ to integer $i$ such that $\sum_{i=1}^{6} s_{i} i \equiv 0 \bmod 13$.

With these sets of differences found and recorded, the next stage was to consider all possible arrangements of the differences and determine which produced 6 -cycles. For example the arrangement $-3,-1,+4,+5,+6,+2$ would not produce a 6 -cycle as the first and fourth elements would be the same, that is, $v_{1}=v_{4}$. Such arrangements were discarded and only those producing 6 -cycles were retained.

The result of the search so far was a collection of 6-cycles each of which could be used to generate a 6-cycle system; however many of these 6-cycles were not 2-perfect. The next step was to determine which 6-cycles would produce 2-perfect systems. This was done by considering the edges of the distance 2 graph, that is, $\left(v_{1}, v_{3}, v_{5}\right)$ and $\left(v_{2}, v_{4}, v_{6}\right)$, and checking to see if this was a Steiner triple system. Now $\left(v_{1}, v_{3}, v_{5}\right)$ and $\left(v_{2}, v_{4}, v_{6}\right)$ generate a Steiner triple system if and only if each of 1 to 6 occurs as the absolute value of a $\delta_{i}$ where $\delta_{i}=v_{i+2}(\bmod \theta)-v_{i}, 1 \leqslant i \leqslant 6$.

From the list of 6-cycles which would generate 2-perfect 6-cycle systems it was a simple matter to generate the systems and from them the quasigroup tables.

\section{COMPARING THE SYSTEMS}

At this stage I checked to see which of the new systems were isomorphic to the system from [2] and then, for those which were not isomorphic to the system from [2], I checked to see which were isomorphic to each other. In order to do this I wrote another computer program.

Mapping from one pair of vertices in a system to a pair of vertices in a second system completely determines the entire mapping from the first system to the second since the corresponding quasigroups are two-generator. This fact was used when writing the program to check for isomorphisms between systems.

The result of the comparisons between systems was the separation of the systems into two classes, one class containing systems isomorphic to the system in [2] and another class containing systems all of which were isomorphic to each other. This showed that there are at most two non-isomorphic cyclic 2-perfect 6-cycle systems of order 13 .

Retaining one system from each class left me with the systems $(1)(0,5,2,8,7,9)+i$ and (2) $(0,11,1,9,10,4)+i, 0 \leqslant i \leqslant 12$, components reduced modulo 13 .

Using the program which checks for isomorphisms between systems, the automorphism groups for these systems were found. Both have the same automorphism group of order 39 with generating elements (0 123456789101112$)$ and (1 39$)(265)(41210)(7811)$. So the standard method of showing non-isomorphism by showing that the automorphism groups are not isomorphic could not be used. 
However, using quasigroup laws did produce the result. Several two-variable laws are known for the quasigroup of system (1) other than the standard laws mentioned earlier. The technique for finding such laws is described in [1]. This technique was used to obtain the following laws:

$$
x \circ y=(c \circ[d \circ(c \circ d)]) \circ([d \circ(c \circ d)] \circ c)
$$

where

$$
\begin{aligned}
& d=(a \circ[b \circ(a \circ b)]) \circ([(b \circ[a \circ b]) \circ a] \circ[a \circ(b \circ[a \circ b])]) \\
& c=(a \circ[b \circ(a \circ b)]) \circ([b \circ(a \circ b)] \circ a) \\
& b=([x \circ y] \circ z) \circ([z \circ(x \circ y)] \circ[(x \circ y) \circ z]) \\
& a=([x \circ y] \circ z) \circ(z \circ[x \circ y]) \\
& z=(x \circ[y \circ x]) \circ([x \circ y] \circ[x \circ(y \circ x)])
\end{aligned}
$$

$$
\begin{aligned}
y & =[g \circ(f \circ g)] \circ[g \circ f] \\
g & =[c \circ(d \circ c)] \circ[(c \circ d) \circ(c \circ[d \circ c])] \\
f & =[c \circ(d \circ c)] \circ[c \circ d] \\
c & =[b \circ(w \circ b)] \circ[(b \circ w) \circ(b \circ[w \circ b])] \\
d & =[b \circ(w \circ b)] \circ[b \circ w] \\
b & =[a \circ(v \circ a)] \circ[(a \circ v) \circ(a \circ[v \circ a])] \\
w & =[a \circ(v \circ a)] \circ[a \circ v] \\
a & =[z \circ(u \circ z)] \circ[(z \circ u) \circ(z \circ[u \circ z])] \\
v & =[z \circ(u \circ z)] \circ[z \circ u] \\
z & =[x \circ(y \circ x)] \circ[(x \circ y) \circ(x \circ[y \circ x])] \\
u & =[x \circ(y \circ x)] \circ[x \circ y] .
\end{aligned}
$$

Law (a) holds in the quasigroup of system (1) but not in the quasigroup of system (2). Consider the substitutions $x=0$ and $y=2$; the left hand side of the equation becomes 6 in system (2) whereas the right hand side of the equation is 0 . Conversely, Law (b) holds in the quasigroup of system (2) and not in the quasigroup of system (1). This time consider the substitutions $x=0$ and $y=1$; the right hand side of the equation becomes 0 which is not equal to $y$.

This proves that the two quasigroups and hence the two systems are not isomorphic. Hence the theorem of this paper is proven.

\section{REFERENCES}

[1] R.A.H. Gower, S. Oates-Williams, D. Donovan and E.J. Billington, 'On the quasigroup 
variety arising from a 2-perfect 6-cycle system of order 13', J. Combin. Math. Combin. Comput. (to appear).

[2] C.C. Lindner, K.T. Phelps and C.A. Rodger, 'The Spectrum for 2-perfect 6-cycle systems', J. Combin. Theory A (to appear).

[3] C.C. Lindner, 'Graph decompositions and quasigroup identities', in Proceedings of the Second International Catania Combinatorial Conference, "Graphs, designs and combinatorial geometries" (Universita di Catania, Catania, Sicily, 1989 to appear).

Department of Mathematics

The University of Queensland

Queensland 4072 